\title{
Stability Analysis of Two Dimensional Fluid Flow in Rayleigh-Bénard Convection Along With Rotation Using DTM-Padé Approximation
}

\author{
H V Gangamani, V Pooja and Achala L Nargund
}

\begin{abstract}
Two dimensional Rayleigh-Bénard convection in a Boussinesq fluid is revisited using DTM-Padé approximation. The stationary and oscillatory instability analysis is obtained using critical Rayleigh numbers. We observe that the effect of increasing the rotational rate is to increase the critical wave number and also the solutions are oscillating more in the horizontal direction as the rotational rate is increased. We have analysed the stability conditions to determine the type of instability at the onset of convection that were dependent on the value of the Taylor number and the Prandtl number. Asymptotic limits reveal that the flow would be comprised of columns of fluid aligned with the rotational axis since as the wave number increases the rotational rate also increases and the critical wave length decreases by setting the onset of convection in the form of tall thin columns. The flow equations are solved to obtain the linearised solutions and Differential Transform Method is applied along with Padé approximation to yield solutions without discretization and linearization and results are displayed in the graphical forms.
\end{abstract}

Index Terms- Rayleigh- Bénard convection, vorticity, Differential Transform Method, DTM- Padé approximation, Stability Analysis.

\section{INTRODUCTION}

Two dimensional Rayleigh-Bénard convection in a Boussinesq fluid is the simplest possible system that exhibits convective instability. Moreover it contains the same basic physics as occurring in many geophysical and astrophysical systems, such as the interiors of the Earth and the Sun. We study this ubiquitous system with and without the effect of rotation, for stress free boundary conditions[1]. Variations are observed for a range of different values of the Rayleigh, Prandtl and Taylor numbers obtained during the process of computation. The rapid rotation of the Earth has a profound effect on the appearance of the convection and can be explained by the Taylor- Proudman theorem. This asserts that motion is uniform in the same direction of the axis of rotation (Taylor (1917), Taylor (1922) and Proudman(1916)). This leads to so-called Taylor columns[4].

Gangamani H V, Post Graduate Department of Mathematics, Bengaluru City University/ MES College of Arts,Commerce and Science/ Mysore Education Society (R), Bengaluru, India, 9148325125.

Pooja V, Post Graduate Department of Mathematics, Bengaluru Central University/ MES College of Arts,Commerce and Science / Mysore Education Society (R), Bengaluru, India.

Achala L. Nargund, Post Graduate Department of Mathematics, Bengaluru City University / MES College of Arts,Commerce and Science / Mysore Education Society (R), Bengaluru, India.
The linear theory of Rayleigh- Bénard convection gives the critical value of the Rayleigh number at which convection onsets. when the analysis is conducted in terms of normal modes, this theory predicts either exponential growth or decay, and gives no further insight into the dynamics after the system has become unstable[2] and the influence of the temperature boundary conditions at the sidewall on the heat transport in Rayleigh-Bénard (RB) convection [3] gives the majority of the convective systems that are found in nature[30] are influenced by rotation, nearly all modern experiments on Rayleigh-Bénard convection incorporated in King et al[5,6,7].

Diaz \& Brevdo [8] obtained for the mixed convection of a Newtonian fluid in porous media using numerical investigations of pattern formation in the classical Rayleigh-Bénard convection with cylindrical geometry in the regime of low Prandtl numbers and moderate aspect ratio and beyond the onset of convection, found straight and bent rolls as stable patterns $[13,14]$ but Mixed convection $[9,10,11]$ has localized instabilities. Numerical simulations of rotating Rayleigh-Bénard convection.[16,17] for transition flow regimes in a rotating Rayleigh-Benard convection with rigorous upper limits on the vertical heat transport $[19,20]$ in two dimensional Rayleigh-Bénard convection between stress-free isothermal boundaries are derived from the Boussinesq approximation[29]. Analytical solutions for both Newtonian and inelastic non-Newtonian fluids with slip boundary conditions are obtained[23]. By increasing the Rayleigh number as in [18] Prandtl and Rayleigh numbers dependences in Rayleigh-Bénard convection is evident in Roche et al [22,24] through conducting experiment in turbulent regimes where the majority of the heat is convected through the layer or cell[25] and pattern selection in viscoelastics fluids [26]

The influences of geometry and physical parameters (Prandtl number, Taylor Number)[21] significance by linear stability[27,28] analysis is focused in this paper. Further along with the understanding of instability mechanisms, the flow equations are solved using Differential Transform method and results are plotted using Graphs.

\section{GEOMETRY AND BOUNDARY CONDITIONS FOR THE FLOW WITH ROTATION}

The fluid can either be confined between two fixed walls, have its upper surface free to the air, or have both surfaces free. The last case is unrealistic, but will be the one considered below because it is the most easily handled analytically.

In either case(fixed wall or free surface) the temperature is held constant along each boundary to give an thermal gradient- $\beta$. 


$$
\begin{array}{cc}
T=T_{0} & z=0 \\
T=T_{1}=T_{0}-\beta d & z=d
\end{array}
$$

The boundary condition on the velocity are as follows. At a fixed wall we have,

$$
u=v=0, \quad \mathrm{~W}=0
$$

At a free surface it can be shown that,

$w=\partial w^{2} / \partial z^{2}=0($ stress free boundary condition $)$

We have,

$$
\begin{gathered}
\quad \nabla . u=0 \\
\frac{\partial u}{\partial t}=-\nabla p+\operatorname{Ra} \operatorname{Pr} \theta Z-T a^{1 / 2} \operatorname{Pr} Z \times u+\operatorname{Pr} \nabla^{2} u \\
\frac{\partial \theta}{\partial t}=\omega+\nabla^{2} \theta
\end{gathered}
$$

where $\omega$ is the $\mathrm{z}$-component of the fluid velocity. In order to eliminate the pressure term we begin by taking curl and double curl of equation

$$
\frac{\partial\left(\nabla^{2} u\right)}{\partial t}=\operatorname{Ra} \operatorname{Pr} \frac{\partial^{2} \theta}{\partial x^{2}} Z-T a^{1 / 2} \operatorname{Pr} \frac{\partial w}{\partial \theta}+\operatorname{Pr} \nabla^{2} u
$$

Velocity is given by $\vec{\omega}=\nabla \times \vec{q}$

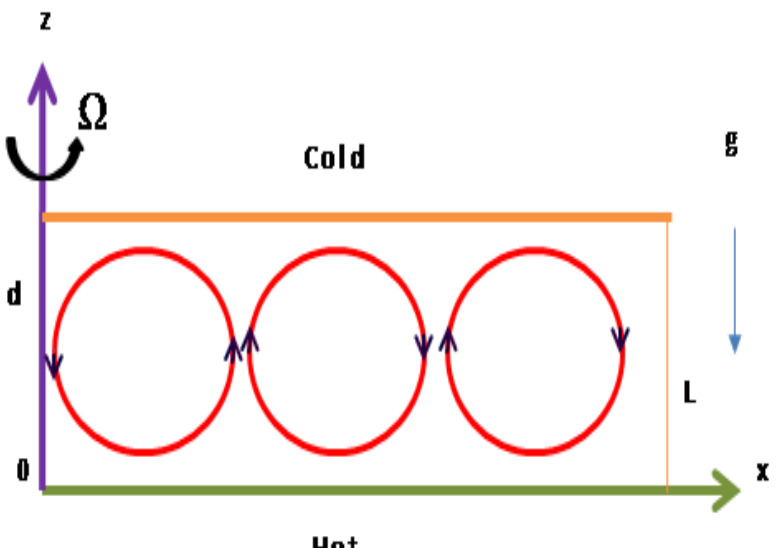

Fig.1. Sketch of the Rayleigh- Benard convection through rotation

We take z-components of these equations, along with temperature equation, as the equations upon which we conduct our linear stability analysis.

$$
\begin{aligned}
& \frac{\partial \zeta}{\partial t}=T a^{1 / 2} \operatorname{Pr} \frac{\partial w}{\partial t}+\operatorname{Pr} \nabla^{2} u \\
& \frac{\partial\left(\nabla^{2} \omega\right)}{\partial t}=\operatorname{Ra} \operatorname{Pr} \frac{\partial^{2} \theta}{\partial x^{2}} Z-T a^{1 / 2} \operatorname{Pr} \frac{\partial \zeta}{\partial t}+\operatorname{Pr} \nabla^{2} \omega \\
& \frac{\partial \theta}{\partial t}=\omega+\nabla^{2} \theta
\end{aligned}
$$

\section{NORMAL MODE ANALYSIS ALONG WITH DTM PADÉ APPROXIMATION}

We now applying normal mode solutions of the form

$$
\begin{aligned}
& w(x, z, t)=\operatorname{Re}\left\{w(z) e^{i a x+\sigma t}\right\} \\
& \zeta(x, z, t)=\operatorname{Re}\left\{Z(z) e^{i a x+\sigma t}\right\} \\
& \theta(x, z, t)=\operatorname{Re}\left\{\Theta(z) e^{i a x+\sigma t}\right\}
\end{aligned}
$$

In the above expansions, the functions $w(z), Z(z)$ and $\Theta(z)$ could be complex. A is the wave number of a particular normal mode and $\sigma$ is the complex Eigen value that shall determine the stability of the system. It is actually the real part of the Eigen value that determines the stability of the system. Note that for $R(\sigma)<0$ as time evolves the solutions will decay exponentially, and hence the system is stable. For $R(\sigma)>0$ as time evolves the solutions will grow exponentially, and hence the system is unstable. The case $R(\sigma)=0$ characterizes the point at which the system is neither stable nor unstable, often termed marginally stable. $\sigma$ can be thought of as a bifurcation parameter, and since the stability of the system changes at $\sigma=0$, this is the corresponding bifurcation point. Hence there are two different classes of instability that may occur: instability due to a purely real Eigen value going through zero - a direct, or ordinary bifurcation and instability due to the real part of a complex conjugate pair of Eigen values going through zero-a Hopf bifurcation. The former is often termed a stationary instability and the latter is often termed over stability, or an oscillatory instability.

Inserting (3.1) into equations (2.9), (2.10) and (2.11) respectively gives

$$
\begin{aligned}
& \sigma Z=T a^{\frac{1}{2}} \operatorname{Pr} D W+\operatorname{Pr}\left(D^{2}-a^{2}\right) Z \\
& \sigma\left(D^{2}-a^{2}\right) W=-a^{2} \operatorname{Ra} \operatorname{Pr} \Theta-T a^{\frac{1}{2}} \operatorname{Pr} D Z+\operatorname{Pr}\left(D^{2}-a^{2}\right)^{2} W \\
& \sigma \Theta=W+\left(D^{2}-a^{2}\right) \Theta
\end{aligned}
$$

Rearranging the above equations so that like terms are together gives

$$
\begin{aligned}
& \left(D^{2}-a^{2}-\frac{\sigma}{\operatorname{Pr}}\right) Z=-T a^{\frac{1}{2}} D W \\
& \left(D^{2}-a^{2}\right)\left(D^{2}-a^{2}-\frac{\sigma}{\operatorname{Pr}}\right) W=a^{2} R a \Theta-T a^{\frac{1}{2}} D Z \\
& \left(D^{2}-a^{2}-\sigma\right) \Theta=-W
\end{aligned}
$$

where $\zeta$ is the $z$-component of vorticity given by, 
along with stressfree boundary conditions,

$W=D^{2} W=0 \quad$ at $z=0$ and $z=1$

$D Z=\Theta=0 \quad$ at $z=0$ and $z=1$

using the appropriate DTM transformations in the set of equations (3.2),(3.3) and (3.4) we have,

$(k+1)(k+2) F(k+2)=\left(a^{2}+\frac{\sigma}{\operatorname{Pr}}\right) F(k)$

$-T a^{1 / 2}(k+1) G(k+1)$

$(k+1)(k+2)(k+3)(k+4) G(k+4)=$

$\left(a^{2}+\frac{\sigma}{\operatorname{Pr}}\right)(k+1)(k+2) G(k+2)-\left(a^{2}+\frac{\sigma}{\operatorname{Pr}}\right) G(k)$

$-(\mathrm{Ta})^{1 / 2}(\mathrm{k}+1) \mathrm{F}(\mathrm{k}+1)-\mathrm{a}^{2} \mathrm{RaH}[\mathrm{k}]$

$(\mathrm{k}+1)(\mathrm{k}+2) \mathrm{H}[\mathrm{k}+2]=-\mathrm{F}(\mathrm{k})+\left(\mathrm{a}^{2}+\sigma\right) \mathrm{H}(\mathrm{k})$

along with boundary conditions,

$\mathrm{H}(0)=0, \quad \mathrm{H}(1)=-1, \quad \mathrm{~F}(0)=0, \quad \mathrm{~F}(0)=0$

$G(0)=0, \quad \mathrm{G}(1)=-1, \quad \mathrm{G}(2)=0, \quad \mathrm{G}(3)=0$

\section{LIMITING FROM ROTATING TO NON-ROTATING CASE}

For rotating convection we have to assume that in general the Eigen values are complex. Enforcing this and converting equation (3.6) to single variable by operating using the operators, $\left(D^{2}-a^{2}-\sigma\right)\left(D^{2}-a^{2}-\frac{\sigma}{\operatorname{Pr}}\right)$ gives

$$
\begin{aligned}
& \left(D^{2}-a^{2}\right)\left(D^{2}-a^{2}-\frac{\sigma}{\operatorname{Pr}}\right)^{2}\left(D^{2}-a^{2}-\sigma\right) W= \\
& a^{2} \operatorname{Ra}\left(D^{2}-a^{2}-\frac{\sigma}{\operatorname{Pr}}\right) W-\operatorname{TaD}^{2}\left(D^{2}-a^{2}-\sigma\right) W
\end{aligned}
$$

Since $W=W_{0} \operatorname{Sin}(n \pi z)$ satisfies the boundary conditions we obtain

$$
\begin{aligned}
& \left(n^{2} \pi^{2}+a^{2}\right)\left(n^{2} \pi^{2}+a^{2}+\frac{\sigma}{\operatorname{Pr}}\right)\left(n^{2} \pi^{2}+a^{2}+\sigma\right) \\
& -a^{2} \operatorname{Ra}\left(n^{2} \pi^{2}+a^{2}+\frac{\sigma}{\operatorname{Pr}}\right)+n^{2} \pi^{2} T a\left(n^{2} \pi^{2}+a^{2}+\sigma\right)=0
\end{aligned}
$$

which is the required Dispersion relation and $\sigma$ is the eigen value. In the limit as $T a \rightarrow 0$ the rotating system converges to the non-rotating system and for $n=1$, equating like powers of $\sigma$ gives,

$$
\sigma^{3}+c_{2} \sigma^{2}+c_{1} \sigma+c_{0}=0
$$

Where the coefficients, $\mathrm{C}_{1}, \mathrm{C}_{2}$ and $\mathrm{C}_{0}$ are given by

$$
\begin{aligned}
& c_{2}=\left(\pi^{2}+a^{2}\right)(1+2 \operatorname{Pr}) \\
& c_{1}=\left(\operatorname{Pr}\left(\pi^{2}+a^{2}\right)^{2}(1+2 \operatorname{Pr})+\frac{\pi^{2} T a \operatorname{Pr}-a^{2} R a}{\left(\pi^{2}+a^{2}\right)}\right) \\
& c_{0}=\operatorname{Pr}^{2}\left(\left(\pi^{2}+a^{2}\right)^{3}+\pi^{2} T a-a^{2} R a\right)
\end{aligned}
$$

Clearly $c_{2}>0$ always holds, since its constituent components are all themselves greater than zero. In general $c_{0}$ and $c_{1}$ are not as they are affected by the values of the other parameters. Congruously, $c_{1} c_{2}-c_{0}$ is not always greater than zero due to the effect of the Rayleigh number.

Consider $R a=0$ in this case $c_{0}, c_{1} c_{2}$ and $c_{1} c_{2}-c_{0}$ will all be greater than zero. Since $c_{0}>0$ this means that there is at least one stable-negative-real root. We shall denote this by $\sigma_{0}<0$ Thus equation (4.3) can be factorized as,

$\left(\sigma-\sigma_{0}\right)\left(\sigma^{2}+2 d \sigma_{1}+d_{0}\right)=0$

with the solutions for the other roots given by,

$$
\sigma=-d_{1} \pm \sqrt{d_{1}^{2}-d_{0}}
$$

expanding the above equation we get,

$$
\sigma^{3}+\left(2 d_{1}-\sigma_{0}\right) \sigma^{2}+\left(d_{0}-2 d_{1} \sigma_{0}\right) \sigma-\sigma_{0} d_{0}=0
$$

Comparing with equation (4.3), we get

$\left(2 d_{1}-\sigma_{0}\right)=c_{2},\left(d_{0}-2 d_{1} \sigma_{0}\right)=c_{1}-\sigma_{0} d_{0}=c_{0}$

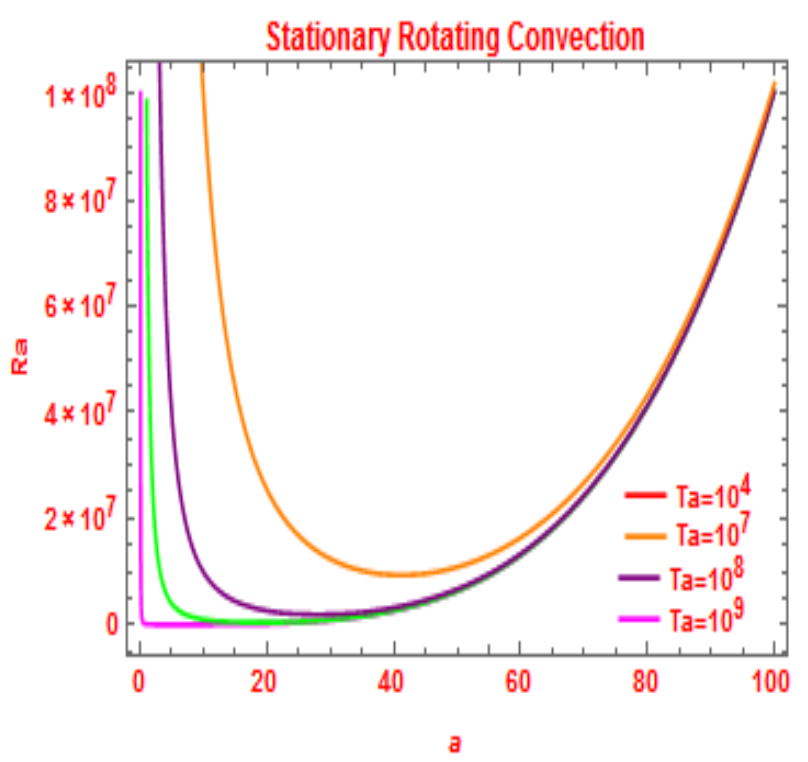

Fig.2. Rayleigh Number verses wave number for different Taylor number.

\section{STATIONARY INSTABILITY ANALYSIS}

For a stationary instability, $c_{0}=0$ giving

$$
R a^{(s)}=\frac{\left(\pi^{2}+a^{2}\right)^{3}+\pi^{2} T a}{a^{2}}
$$

Differentiating equation (5.1) with respect to ' $a$ ' and equating the resultant derivative to zero gives, 


$$
2 a^{6}+3 \pi^{2} a^{4}=\pi^{6}+\pi^{2} T a
$$

We must solve this equation for a for a given Ta in order to find the critical wave number.

\begin{tabular}{|l|l|l|}
\hline $\mathrm{Ta}$ & $a_{c}^{(s)}$ & $R a_{c}^{(s)}$ \\
\hline $10^{4}$ & 5.69797 & $5.377 \times 10^{3}$ \\
\hline $10^{5}$ & 8.62611 & $2.131 \times 10^{4}$ \\
\hline $10^{6}$ & 12.8632 & $9.222 \times 10^{4}$ \\
\hline $10^{7}$ & 19.0244 & $4.147 \times 10^{4}$ \\
\hline $10^{8}$ & 28.0238 & $1.187 \times 10^{6}$ \\
\hline
\end{tabular}

For a given Taylor number, for stationary rotating convection, asymptotic limit $\quad T a \rightarrow \infty \quad$ The dominant balance in this case will be between the term on left hand side and term involving the Taylor number on the right hand side.

$$
2 a^{6} \sim \pi^{2} T a
$$

A. we get an asymptotic solution for $\mathrm{Ra}^{(s)}$ Hence as $T a \rightarrow \infty$, we have,

$$
\begin{gathered}
a_{c}^{(s)} \sim 3\left(\frac{\pi^{2}}{2}\right)^{1 / 6} T a^{1 / 6} \\
R a_{c}^{(s)} \approx 3\left(\frac{\pi^{2}}{2}\right)^{2 / 3} T a^{2 / 3}
\end{gathered}
$$

\section{OSCILLATORY INSTABILITY ANALYSIS}

For a stationary instability, $c_{1} c_{2}-c_{0}=0$ giving

$$
R a^{(o)}=\frac{2(1+\operatorname{Pr})}{a^{2}} \frac{\left((1+\operatorname{Pr})^{2}\left(\pi^{2}+a^{2}\right)^{3}+\pi^{2} T a \operatorname{Pr}^{2}\right)}{(1+\operatorname{Pr})^{2}}(6.1)
$$

To find the critical value once again differentiating and equating to zero we obtain,

$$
2 a^{6}+3 \pi^{2} a^{4}=\pi^{6}+\frac{\pi^{2} T a \operatorname{Pr}^{2}}{(1+\operatorname{Pr})^{2}}
$$

Solving gives the values of ' $a$ ', at $\operatorname{Pr}=0.1$.

\begin{tabular}{|l|l|l|}
\hline $\mathrm{Ta}$ & \multicolumn{1}{|c|}{$a_{c}^{(o)}$} & $R a_{c}^{(o)}$ \\
\hline $10^{4}$ & 2.54236 & $1.731 \times 10^{3}$ \\
\hline $10^{5}$ & 3.58352 & $3.401 \times 10^{4}$ \\
\hline $10^{6}$ & 5.50091 & $1.062 \times 10^{4}$ \\
\hline $10^{7}$ & 8.340066 & $4.161 \times 10^{4}$ \\
\hline $10^{8}$ & 12.4495 & $1.785 \times 10^{6}$ \\
\hline
\end{tabular}

For a given Taylor number, for Oscillatory rotating convection, asymptotic limit $\quad T a \rightarrow \infty \quad$ The dominant balance term involving the Taylor number on the right hand side is given by,

$$
2 a^{6} \approx \frac{\pi^{2} T a \operatorname{Pr}^{2}}{(1+\operatorname{Pr})^{2}}
$$

we get an asymptotic solution for $R a^{(o)}$ Hence as $T a \rightarrow \infty$, we have,

$$
a_{c}{ }^{s} \approx\left(\frac{\pi^{2} \operatorname{Pr}^{2}}{2(1+\operatorname{Pr})^{2}}\right)^{1 / 6} T a^{1 / 6}
$$

$R a_{c}^{\left({ }^{(o)}\right.} \approx 6(1+\operatorname{Pr})\left(\frac{\pi^{2} \operatorname{Pr}^{2}}{2(1+\operatorname{Pr})^{2}}\right)^{2 / 3} T a^{2 / 3}$

which is similar as we obtained during stationary instability

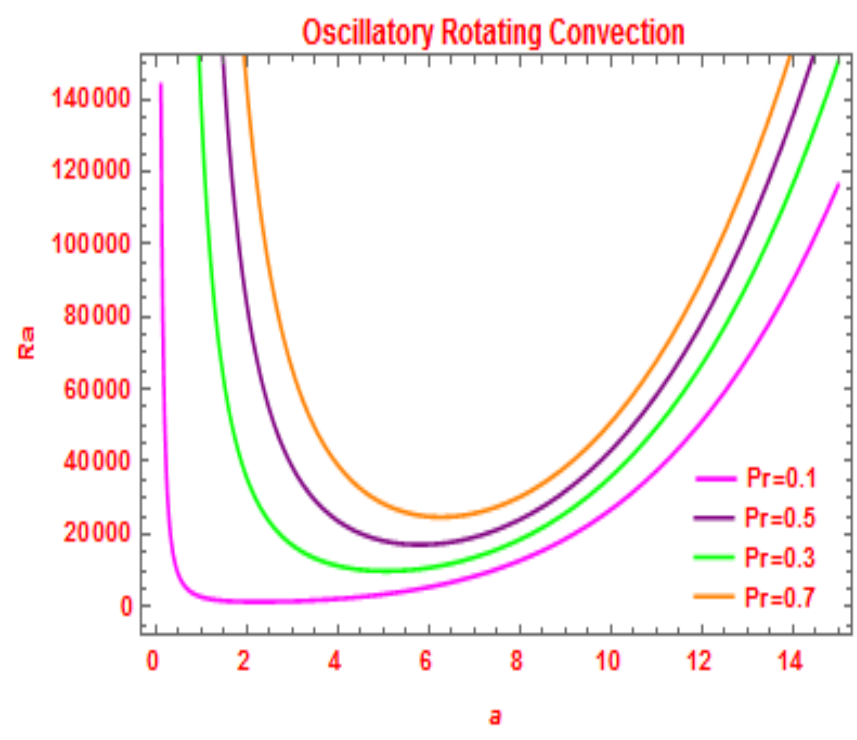

Fig3. Rayleigh Number verses wave number for $\mathrm{Ta}=10^{\wedge} 4$ for Oscillatory Rotatory convection.

The critical number $\operatorname{Pr}_{\mathrm{c}}$ happens when $\mathrm{Ra}_{\mathrm{c}}{ }^{(\mathrm{s})}$ and $\mathrm{Ra}_{\mathrm{c}}{ }^{(\mathrm{o})}$ are equal in the limit $\mathrm{Ta} \rightarrow \infty$ which is given by $\operatorname{Pr}_{\mathrm{c}}=0.676605$. Therefore when $\operatorname{Pr}>\operatorname{Pr}_{c}$ and $\operatorname{Ra}_{c}{ }^{(0)}<\operatorname{Ra}_{c}{ }^{(s)}$ so convection will be stationery and for $\operatorname{Pr}<\operatorname{Pr}_{c}$ for Particular Ta when $\operatorname{Ra}_{\mathrm{c}}{ }^{\left({ }^{\circ}\right)}$ $\mathrm{Ra}_{\mathrm{c}}{ }^{(\mathrm{s})}$ Oscillatory convection will be set.

\section{EIGEN FUNCTION SOLUTIONS}

The observable difference in increasing the rotation rate is to increase the critical wave number [6]. Physically, this means that solutions will oscillate more in the horizontal direction, as the rotation rate is increased. The solution for vertical velocity is $W \sim \sin (n \pi z)$ with mode $\mathrm{n}=1$ facilitating the lowest Rayleigh number. It was necessary for to be of the same parity as $W$ and $Z$ to be of opposite parity. 
Using equations (3.5), (3.6) and (3.7), the solutions for $W, \Theta$ and $Z$ are given by, is a constant determining the amplitude of the perturbations.

$$
\begin{gathered}
W=W_{0} \sin \pi z \\
\theta=\frac{W_{0}}{\left(a^{2}+\pi^{2}\right)} \sin \pi z \\
Z=\frac{\pi T a^{1 / 2} W_{0} \cos \pi z}{\left(a^{2}+\pi^{2}\right)}
\end{gathered}
$$

W $y$ gyre, $W_{0}$ Using the original form of normal mode expansions given by equations (5.5),(5.6) and (5.7), we have,

$$
\theta=\cos a x \sin \pi z
$$

$\omega=\left(a^{2}+\pi^{2}\right) \cos a x \sin \pi x$

$\zeta=\pi T a^{1 / 2} \cos a x \sin \pi z$

Where, $W_{0}=a^{2}+\pi^{2}$ is arbitrarily-chosen.

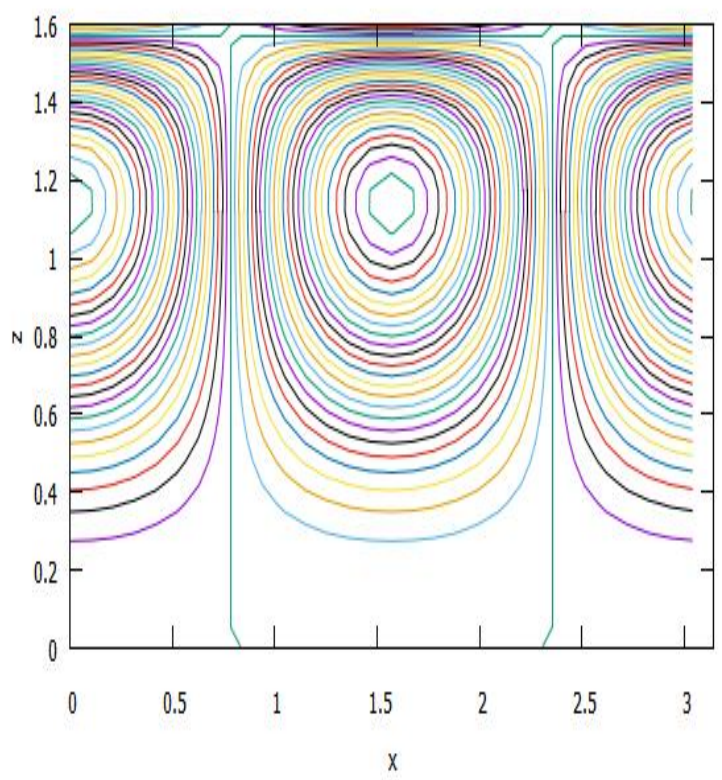

Fig 4. Plots of vertical velocity for $\mathrm{Ta}=10^{4}, \mathrm{Pr}=1$ $R=R a_{c}^{(s)}$, using DTM- Padé Approximation

\section{RESULTS AND DISCUSSIONS}

The fluid flow equations for Two dimensional Rayleigh-Benard convection in a Boussinesq fluid for vertical velocity and Dispersion relation is derived. The graph of Rayleigh Number verses wave number is plotted in figure 2. In figure 2 we see that the curves are oriented towards the rotational axis as Rayleigh number increases for different Taylor number. The Plots of vertical velocity, vorticity and temperature are plotted in figures 4, figures 5 and figure 6 using Differential Transform Method along with Padé
Approximation where convection cell formation can be visualized.

The linearised solutions are plotted in figures 6 , figures 7 and figure 8 respectively. It can be visualized that the flow is composed of rising and sinking columns of fluid, that are driven by the buoyancy. These are known as convection cells. For a larger Taylor number, in a box of the same length, the linear Eigen functions would be composed of more cells in the horizontal direction

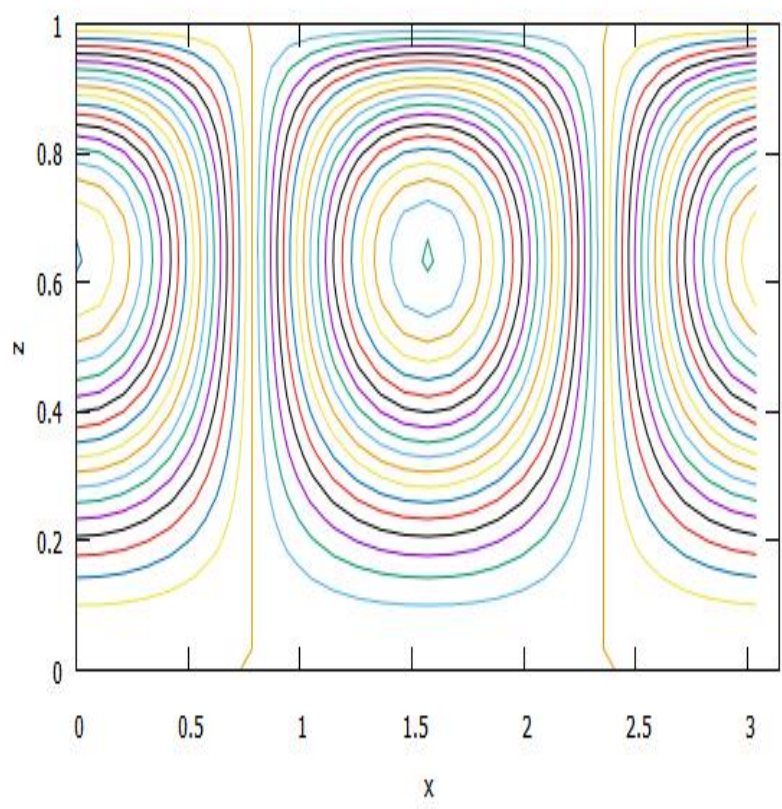

Fig 5. Plots of vertical vorticity for $\mathrm{Ta}=10^{4}, \mathrm{Pr}=1$ $R=R a_{c}^{(s)}$, using DTM- Padé Approximation.

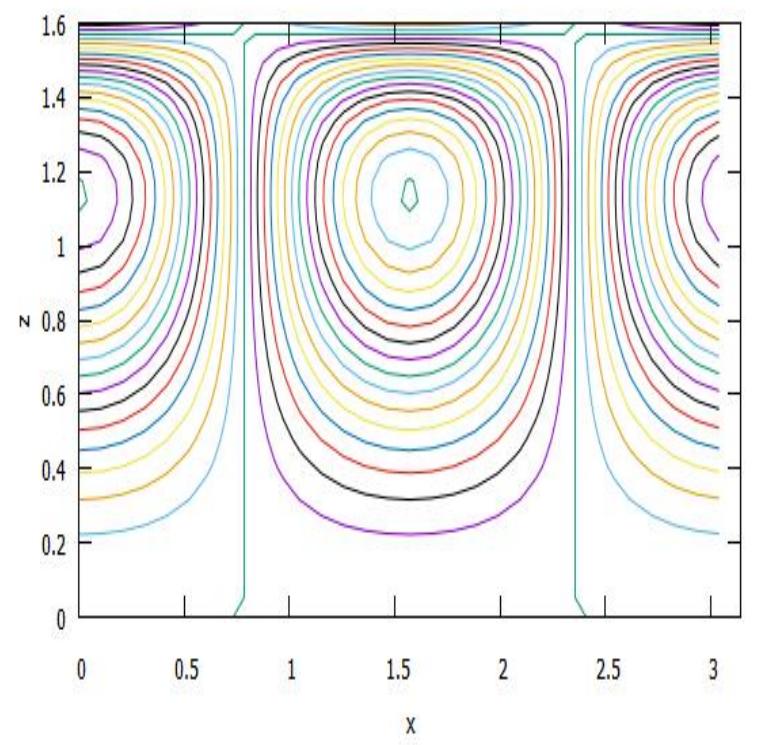

Fig 6. Plots of vertical temperature for $\mathrm{Ta}=10^{4}$, $\operatorname{Pr}=1, R=R a_{c}^{(s)}$ using DTM-Padé Approximation. 


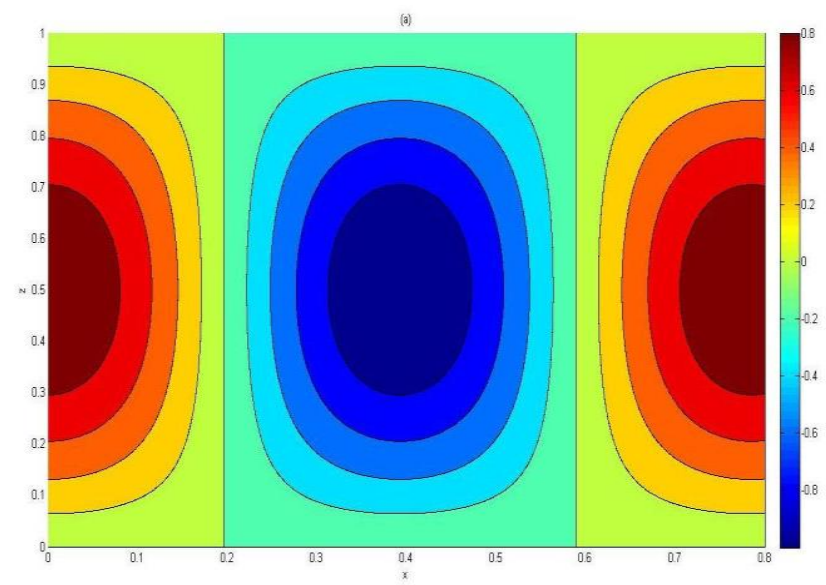

Fig 7. Plots of vertical velocity for $\mathrm{Ta}=10^{4}, \mathrm{Pr}=1$, $R=R a_{c}^{(s)}$, using Matlab.

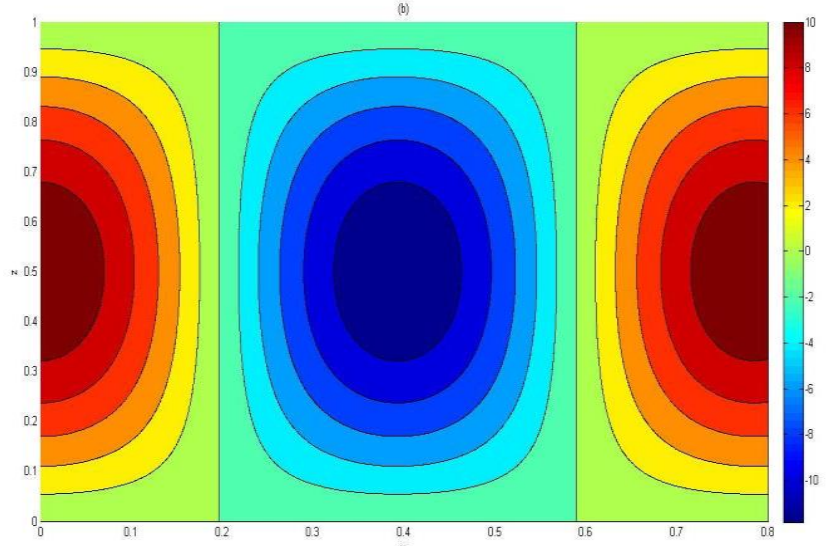

Fig 8. Plots of vertical vorticity for $\mathrm{Ta}=10^{4}, \mathrm{P}=\mathrm{Pr}$ $R=R a_{c}^{(s)}$, using Matlab

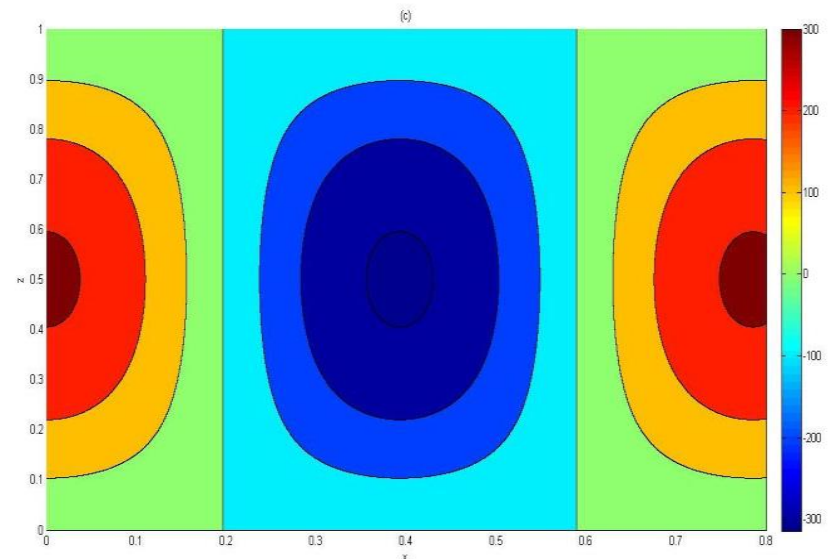

Figure 9. Plots of vertical vorticity for $\mathrm{Ta}=10^{4}, \mathrm{Pr}=1$ $R=R a_{c}^{(s)}$, using Matlab

\section{CONCLUSIONS}

The flow system for two dimensional Rayleigh-Bénard convection in a Boussinesq fluid is studied to find the critical value of the Rayleigh number, for a particular wave number, at which convection could occur. for the Linear theory for the rotating system. Furthermore, we distinguished between the type of instability at the onset of convection-stationary or oscillatory. We computed stability conditions to determine the type of instability at the onset of convection that were dependent on the value of the Taylor number and the Prandtl number and also by solving the linearised equations using Differentia transform method to analyses the solutions obtained.

\section{ACKNOWLEDGMENT}

The study is supported by VGST GRD 105 Government of Karnataka. We also thank Mysore Education Society(R ).

\section{APPENDIX}

TABLE 1: THE OPERATIONS FOR THE ONE-DIMENSIONAL DTM.

\begin{tabular}{|l|l|}
\hline Original Function & Transformed Function \\
\hline$w(x)=u(x) \pm v(x)$ & $W(x)=\mathrm{U}(x) \pm V(x)$ \\
\hline $\mathrm{W}(x)=\lambda u(x)$ & $\begin{array}{l}W(x)=\lambda U(x) \\
\text { where } \lambda \text { is a constant }\end{array}$ \\
\hline $\mathrm{w}(\mathrm{x})=\frac{d u(x)}{d x}$ & $\mathrm{~W}(\mathrm{k})=(k+1) U(k+1)$ \\
\hline $\mathrm{W}(\mathrm{x})=\frac{d^{r} u(x)}{d x^{r}}$ & $\mathrm{~W}(\mathrm{k})=(k+1)(k+2) \ldots(k+r)$ \\
$\mathrm{W}(\mathrm{x})=u(x) v(x)$ & $\mathrm{W}(k+r)$ \\
\hline $\mathrm{W}(\mathrm{x})=\frac{d u(x)}{d x} \frac{d v(x)}{d x}$ & $\mathrm{~W}(\mathrm{k})=\sum_{\mathrm{r}=0}^{\mathrm{k}} U(r) V(k-r)$ \\
\hline $\mathrm{w}(\mathrm{x})=u(x) \frac{d v(x)}{d x}$ & $\mathrm{~W}(\mathrm{k})=\sum_{\mathrm{r}=0}^{\mathrm{k}}(k(k-r+1)(k-r+1)$ \\
\hline $\mathrm{w}(\mathrm{x})=u(x) \frac{d^{2} v(x)}{d x^{2}}$ & $\mathrm{~W}(\mathrm{k})=\sum_{\mathrm{r}=0}^{\mathrm{k}}(k-r+2)(k-r+1) V(k-r+2)$ \\
\hline
\end{tabular}

\section{REFERENCES}

[1] P.S. Ghoshdastidar, Heat Transfer, Oxford University press, 2004.

[2] Getling A.V. ,Institute of Nuclear Physics, the convective currents in a horizontal layer of fluid when the higher temperature is on the under side,Phil.Mag. 2012 ,32,529-546

[3] Richard J. A. M. Stevens, Detlef Lohse and Roberto Verzicco, Sidewall effects in Rayleigh_Bénard convection, J. Fluid Mech, 2014, vol. 741, pp 1-27, Doi: https://doi.org/10.1017/jfm.2013.664

[4] Gillet, N. and jones, C.A.,The quazi-geostrophic model for rapidly rotating s[herical convection outside the tangent cylinder, J. Fluid Mech, .,2006, vol.554,334-369.

[5] Liu, Y., \& Ecke, R. E. ,Heat transport measurements in turbulent rotating Rayleigh-B'enard convection. Physical Review E, 2009, 80, 036314.

[6] King,E.M.,Stellmach,S,Noir J.,Hansen U., \& Aurnou, J.M.,) Boundary layer control of rotating convection systems. Nature, 2009, 457, 301-304.

[7] King,E.M.,Stellmach,S., \&Aurnout,Heat Transfer by rapidly rotating Rayleigh-Bénard convection, J. Fluid Mech, , 2012, 691, 568-582. Doi: https://doi.org/10.1017/jfm.2011.493 
[8] Diaze E. \& Brevdo, L. ,Absolute/convective instability dichotomy at the onset of convection in a porous layer with either horizontal or vertical solutal and horizontal through flow. J. Fluid Mech., 2011, 681, 567-596.

[9] Grandjean, E. \& Monkewita, P. A., Experimental investigation into localized instabilities of mixed Rayleigh-Bénard-Poiseuille convection. J. Fluid Mech, 2009, 640, 401-419.

[10] Hirata S. C. \& Ouarzazi, M. N., Three-dimensional absolute and convective instabilities in mixed convection of a viscoelastic fluid through a porous medium. Phys. Lett., 2010, A 374 (26), 2661-2666. Doi:https://doi.org/10.1016/Physleta.2010.04.039

[11] Huerre, P. \& Monkewitz, P. A., Local and global instabilities in spatially developing flows. Annu. Rev. Fluid Mech., 1990, 22 (1), 473-537

[12] Kolodner, P., Oscillatory convection in viscoelastic DNA suspensions. J. Non-Newtonian Fluid Mech., 1998,75 (2-3), 167-192

[13] Nicolas, X., Mojtabi, A. \& Platten, J. K. ,Two-dimensional numerical analysis of the Poiseuille-Bénard flow in a rectangular channel heated from below. Phys. Fluids, 1997 9, 337-348.

[14] Rudiger, S. \& Feudel, F., Pattern formation in Rayleigh- Bénard convection in a cylindrical container. Phys. Rev. E, 2000, 62, 4927-4931.

[15] Shraiman, B. I. \& Siggia, E. D., Heat transport in high-Rayleigh-number convection. Phys. Rev. , 1990, A 42 (6), 3650-3653

[16] Rotvig, J., \& Jones, C.A.,. Rotating convection-driven dynamos at low Ekman number. Physical Review E, 2002, 66, 056308. Doi:https://doi.org/10.10103/PhysRevE.66.056308.

[17] Schmitz, S., \& Tilgner, A.,. Transitions in turbulent rotating Rayleigh-B'enard convection. Geophysical and Astrophysical Fluid Dynamics, 2010,104, 481-489

[18] Vincent, A.P., \& Yuen, D.A., Plumes and waves in two-dimensional turbulent thermal convection. Physical Review E, 1999, 60, 2957-2963.

[19] Whitehead, J.P., \& Doering, C.R, Ultimate State of Two-Dimensional Rayleigh-Bénard Convection between Free-Slip Fixed-Temperature Boundaries. Physical Review Letters, 2011,106, 244501. Doi:https://doi.org/10.10103/PhysRevLett.106.244501

[20] Hebert, F., Hufschmid, R., Scheel, J. \& Ahlers, G. ,Onset of Rayleigh-Bénard convection in cylindrical containers. Phys. Rev. , 2010,E 81, 046318

[21] Roche, P. E., Castaing, B., Chabaud, B. \& Hebral, BPrandtl and Rayleigh numbers dependences in Rayleigh-Bénard convection. Europhys. Lett., . 2002 , 58, 693-698

[22] Scheel, J. D., Kim, E. \& White, K. R.,Thermal and viscous boundary layers in turbulent Rayleigh-Bénard convection. J. FluidMech.,2012,711,281-305.Doi: https://doi.org/10.1017/jfm.2012. 392.

[23] Ferras, L. L., Nobrega, J. M. \& Pinho, F. T, Analytical solutions for Newtonian and inelastic non-Newtonian flows with wall slip. J. Non-Newtonian Fluid Mech., . 2012, 175-176, 76-88. Doi:https://doi.org/10.1016i.jnnfm.2012.03.004

[24] Mokarizadeh, H., Asgharian, M. \& Raisi, A., Heat transfer in Couette-Poiseuille flow between parallel plates of the Giesekus viscoelastic fluid. J. Non-Newtonian Fluid Mech., 2013, 196, 95-101 Doi:https://doi.org/10.1016J.JNNFM.2013.01.007

[25] Nan Shi, Mohammad S. Emran and Jörg Schumacher, A. Boundary layer structure in turbulent Rayleigh-Bénard convection. J. FluidMech.,2012,706,55-33, Doi:https://doi.org/10.1017/jfm.2012.20 7.

[26] Albaalaki, B. \& Khayat, R. ,Pattern selection in the thermal convection of non-Newtonian fluids. J. Fluid Mech. (2011) 668, 500-550.

[27] David Sonadak, Leslie M. Smith and Fabian Waleffe, (2015), Optimal heat transport solutions for Rayleigh-Bénard convection,. J. Fluid Mech. 784, 565-595.,Doi: https://doi.org/10.1017/jfm.2015.615
[28] Deardorff, J.W. (1970) Convective Velocity and Temperature Scales for the Unstable Planetary Boundary Layer and for Rayleigh Convection. Journal of the Atmospheric Sciences, 27, 1211-1213.

[29] Gilman, P.A. (1977), Nonlinear Dynamics of Boussinesq Convection in a Deep Rotating Spherical Shell I. Geophysical and Astrophysical Fluid Dynamics, 8, 93-135.

[30] Hollerbach, R., \& Jones, C.A. (1993b). Influence of the Earth's inner core on geomagnetic fluctuations and reversals. Nature, $365,541-543$.

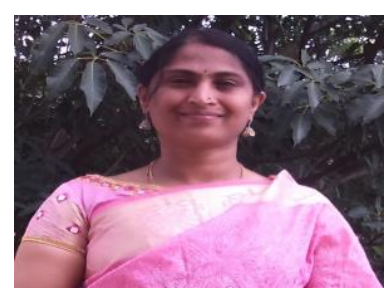

Dr. H.V. Gangamani born in Bangalore, Karnataka, India on 23rd August 1975 has obtained M. Sc. securing Fourth Rank and successive Ph. D degree in the year 2009 for her work on Internal Gravity Wave Tunneling in conducting and non-conducting stratified flows from Bangalore University, Bangalore, India under the able guidance of Prof. M. Venkatachalappa.. FNA, FNASc, FISET. She has also done her Post Doctoral studies from the Indian Institute of Science, Bangalore as RASS Research Associate in COAS, She has participated in 21 Conferences and presented papers in 21 National and International Conferences. Presently She is working as an Assistant Professor and guided 18 M. Sc. Students for the Project Work in Applied Mathematics. The topics of research interest are Fluid Dynamics, Applied Mathematics, Atmospheric Science, Numerical and Semi-Analytical Techniques.

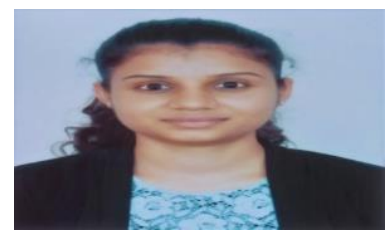

Pooja V, born in Karnataka, India, M. Sc, Post graduate student of Bengaluru Central University, having completed Bachelor of Science in Electronics course from the Bengaluru Central University, Karnataka, has also completed courses in Electronic Product Design and Applications and is quick learner and has ability to deal with solving comprehensive problems is working as assistant Professor in T John College of Engineering. She was also involved in the Project work during the course of her study in the Post graduate Department of Mathematics and Research Center in Applied Mathematics, entitled "Study of Convection Using DTM-Pade" Approximation for Thermal Boundary Layer". She has also participated in workshops and conferences during her tenure in the Post Graduate Department of Mathematics.

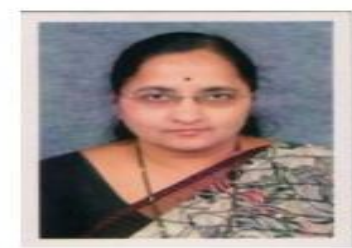

Prof. Achala. L. Nargund born in Gulbarga, Karnataka, India on 11th January 1960 received her doctorate in Applied Mathematics in 2001 from Bangalore University, Bangalore, India. Since 1992 working at P. G. Department of Mathematics, MES College, Bangalore, Karnataka, India. She has delivered many invited talks at conferences and seminars. She has attended and presented papers in 15 National and International Conferences. 9 students have obtained Doctorate degree under her Guidence. She is now guiding 6 students for $\mathrm{Ph}$. D and also has guided 10 students for M. Phil. She is involved in Research in Fluid Dynamics, Nonlinear differential equations, Biomechanics, and Numerical analysis. 